\title{
Uptake and transport of pullulan acetate nanoparticles in the BeWo b30 placental barrier cell model
}

This article was published in the following Dove Press journal:

International Journal of Nanomedicine

Hongbo Tang,' Ziwen Jiang, Haibo $\mathrm{He},{ }^{3}$ Xiaoqin $\mathrm{Li}^{3}$ Haipeng Hu,' Ning Zhang,' Yinmei Dai, ${ }^{2}$ Zhimin Zhou ${ }^{4}$

'Department of Pharmacy, Beijing Obstetrics and Gynecology Hospital, Capital Medical University, Beijing I00006, People's Republic of China; ${ }^{2}$ Department of Gynecology, Beijing Obstetrics and Gynecology Hospital, Capital Medical University, Beijing 100006, People's Republic of China; ${ }^{3}$ College of Biological and Pharmaceutical Sciences, China Three Gorges University, Yichang 443002, People's Republic of China; ${ }^{4}$ Biomedical Barriers Research Center, Institute of Biomedical Engineering, Chinese Academy of Medical Sciences \& Peking Union Medical College, Tianjin Key Laboratory of Biomedical Materials, Tianjin, 300192, People's Republic of China

Correspondence: Yinmei Dai Department of Gynecology, Beijing Obstetrics and Gynecology Hospital, Capital Medical University, No 17, Qi He Lou Street, Dongcheng District, Beijing 100006, People's Republic of China $\mathrm{Tel} / \mathrm{fax}+86 \quad 10$ 859। 5110 Email fcyydym@।63.com

Zhimin Zhou

Biomedical Barriers Research Center, Institute of Biomedical Engineering, Chinese Academy of Medical Sciences \& Peking Union Medical College, Tianjin Key Laboratory of Biomedical Materials, 236 Baidi Road, Nankai District, Tianjin 300192, People's Republic of China $\mathrm{Tel} / \mathrm{fax}+862287890868$

Email zhouzhiminf@126.com
Introduction: Nanomedicine has shown a great potential in perinatal medicine because of its characteristics of sustained, controlled release and targeting ability; on the other hand, it may also lead to unexpected toxicities such as embryotoxicity and even malformation after crossing the placental barrier, but data concerning transplacental transport are scarce. Pullulan acetate (PA) nanoparticles (NPs) are a promising nanocarrier derived from natural polysaccharide; however, their transplacental transport ability and mechanism are unknown.

Materials and methods: In this study, fluorescein isothiocyanate (FITC) conjugated PA (PAFITC) was synthesized. PA-FITC NPs were characterized by dynamic light scattering, transmission electron microscopy (TEM) and scanning electron microscopy (SEM). The cytotoxicity of PAFITC NPs at concentrations of $15,30,60,125,250,500,1,000$ and 2,000 $\mu \mathrm{g} / \mathrm{mL}$ was studied by cell counting kit-8. The human chorionic gonadotrophin (HCG) cytokine assay was conducted to evaluate the biological function of BeWo b30 cells. Endocytic mechanisms of PA-FITC NPs were investigated via fluorescence analysis. The monolayer properties were characterized by TEM, tight junction staining, transepithelial electrical resistance and fluorescein sodium transportation. The transport ability was measured in the cell based transwell model by confocal imaging and SEM. Results: PA-FITC NPs were almost spherical shape with a size range of 200-300 nm. Cell viability of BeWo b30 cells was up to $100 \%$ in all groups. The concentrations of HCG increased with increasing numbers of cells and culture time, which showed the good biological function of BeWo b30 cells. PA-FITC NPs were rapidly endocytosed through caveolae-mediated endocytosis and pinocytosis, with uptake inhibition rates with nystatin (NY) and colchicines (Col) of $55 \%$ and $51 \%$ respectively. BeWo b30 cell monolayer was formed over 5 days. PA-FITC NPs were found in the cytoplasm of cells on the transwell membranes; while some NPs were found in the basolateral (fetal) compartment over $24 \mathrm{~h}$.

Conclusion: In summary, PA-FITC NPs are nontoxic, can cross the blood-placental barrier, and show mainly internalization to BeWo b30 cells through caveolae-mediated endocytosis and pinocytosis pathways, major via the former pathway. The results could benefit the adjustment and control of the transplacental transport of nanomedicines.

Keywords: transport, embryotoxicity, endocytosis, nystatin, caveolae

\section{Introduction}

Pharmaceutical nanotechnology has generated breakthrough developments in the life science field. ${ }^{1}$ The use of nanoparticles (NPs) for diagnostic and therapeutic applications is generating more and more concern. ${ }^{2}$ Nanomedicines show a great potential to specifically treat maternal, fetal, or placental disorders; on the other hand, it is necessary to prevent embryonic/fetal toxicity and reduce side effects. The biosafety and 
transplacental transport mechanism of NPs are the keys for safe use in pregnant women.

The mechanisms of placental exchange include passive and facilitated diffusion, via transtrophoblastic channels and active transport, such as via endocytosis. ${ }^{3,4}$ Endocytosis may be an important mechanism for translocation of certain types of NP across the maternal-fetal barrier. Macropinocytosis, clathrin-dependent, caveolae-dependent, or clathrin- and caveolae-independent endocytosis, is proposed as the subtype of endocytosis. ${ }^{4,5}$ Macromolecules such as NPs typically enter the placenta via other mechanisms - predominantly pinocytosis/endocytosis and phagocytosis, which depend on the molecules concerned. ${ }^{6}$ The BeWo, Jar, and JEG-3 cell lines have all been used to study the transplacental transfer of a variety of drugs and compounds. ${ }^{7}$ The human, choriocarcinoma-derived BeWo cell line can be a good in vitro model system to investigate the transcellular transport of multiple nutrients and compounds. ${ }^{8,9}$ Ali et al ${ }^{10}$ investigated the transplacental transport of dexamethasone-loaded poly(lactic-co-glycolic acid) (PLGA) NPs using a BeWo (clone b30) cell model. The study found a tenfold increase in permeability of dexamethasone from the apical (maternal) to the basolateral (fetal) side when encapsulated within strongly anionic PLGA NPs. It was also noted that the permeability of the PLGA NPs in BeWo cells was halved when their size increased from 143 to $196 \mathrm{~nm}$. Lopalco et al ${ }^{11}$ prepared oxcarbazepine-loaded NPs from the biocompatible polymer PLGA with or without surfactant and PEGylated PLGA. Transport studies using fluorescent-labeled NPs (loaded with coumarin-6) demonstrated increased permeability of surfactant-coated NPs. Cartwright et $\mathrm{al}^{8}$ studied the transport of 50-nm and 100-nm diameter particles using the in vitro BeWo cell model to determine the nontoxic concentrations of fluorescent polystyrene NPs. Their results demonstrated the transcellular transport of these particles from the apical to the basolateral compartment. Over the course of 24 hours, the apparent permeability across BeWo cells grown on polycarbonate membranes $(3.0 \mu \mathrm{m}$ pore size) was four times higher for the 50-nm particles compared with the $100-\mathrm{nm}$ particles. Transport studies revealed significantly increased permeability of digoxin-loaded NPs across BeWo cell layers compared with free digoxin. P-glycoprotein inhibition also increased the permeability of digoxin, but not digoxin-loaded NPs. This study provided a novel approach to the treatment of fetal diseases such as cardiac arrhythmia. ${ }^{12}$ Grafmueller et $\mathrm{al}^{13}$ analyzed the bidirectional transfer of plain and carboxylatemodified polystyrene particles ranging in size between 50 and $300 \mathrm{~nm}$ using the ex vivo human placental perfusion model. This study indicated that the main translocation mechanism is likely to involve an active, energy-dependent transport pathway. In another study, the exposure of pregnant rodents provided important information on the biodistribution of NPs, including translocation and toxicity to the fetus. ${ }^{14}$ In summary, data on NPs crossing the placental barrier are sparse, especially concerning the transport mechanism. Several recent reviews and government reports have highlighted this as an area in need of more research. ${ }^{3}$

Pullulan has been used as a nanodrug delivery system due to its nonimmunogenic, nontoxic, noncarcinogenic, and nonmutagenic properties. ${ }^{15}$ In our previous studies, ${ }^{16}$ pullulan acetate NPs (PA NPs) and epirubicin-loaded PA NPs (PA/EPI) were prepared, and their biodistribution and safety properties were evaluated. PA NPs were almost spherical in shape with a size range of 200-450 $\mathrm{nm}$ and low $\zeta$ potentials both in distilled water and in $10 \%$ fetal bovine serum. The storage stability of PA NPs was observed in distilled water. PA NPs were stored for at least 2 months with no significant changes in size or $\zeta$ potential. PA NPs were well tolerated at the dose of $200 \mathrm{mg} /$ $\mathrm{kg}$ in mice in a single-dose toxicity test. Moreover, the in vivo pharmacokinetics of PA/EPI was investigated through tail vein injection in rats of $10 \mathrm{mg} / \mathrm{kg}$. Compared with free EPI, $\mathrm{PA} / \mathrm{EPI}$ exhibited higher plasma drug concentrations, longer half-life $\left(\mathrm{t}_{1 / 2}\right)$, and a larger area under the curve.

In this study, we hypothesized that PA NPs would be suitable nanocarriers for use during pregnancy and could be prevented from crossing the placenta. We synthesized and characterized fluorescein isothiocyanate (FITC)-conjugated PA (PA-FITC). PA-FITC NPs were prepared by the dialysis method and characterized by transmission electron microscopy (TEM), size distribution, and $\zeta$ potential techniques. We conducted an extensive evaluation of PA-FITC NP cytotoxicity in a BeWo b30 model. Possible mechanisms involving caveolae, clathrin, macropinocytosis, and pinocytosis were investigated. Human chorionic gonadotropin (HCG) cytokine assay was used to elucidate placental cell functionality. The growth of BeWo b30 cells for NP transport studies was characterized in polycarbonate (PC) Transwell ${ }^{\circledR}$ inserts, and the barrier properties were investigated by TEM, tight junction staining, transepithelial electrical resistance (TEER), and fluorescein sodium (Na-Flu) transport. Following the determination of nontoxic concentrations of fluorescent PAFITC NPs, their transport ability and possible mechanism were measured using the in vitro BeWo cell model.

\section{Materials and methods Materials}

Transwell plates, $\mathrm{PC}$ with $3 \mu \mathrm{m}$ pore size, and the Millicell electrical resistance system (ERS) were purchased from 
Millipore Corp. (Bedford, MA, USA). HCG enzyme-linked immunosorbent assay (ELISA) kits were purchased from Cloud-Clone Corp. (Houston, TX, USA). Cell Counting Kit-8 was purchased from Dojindo Laboratories (Kumamoto, Japan). The choriocarcinoma cell line BeWo b30 was donated by Professor Erik Rytting of University of Texas Medical Branch (Galveston, TX, USA). Pullulan (molecular weight $[\mathrm{MW}]=200,000$ ) was purchased from the Hayashibara Company (Okayama, Japan), and $10 \%$ (volume/volume [v/v]) fetal bovine serum, Hank's balanced salt solution, and DMEM-F12 were purchased from Invitrogen (Thermo Fisher Scientific, Waltham, MA, USA). Phalloidin, chlorpromazine (CPZ), nystatin (NY), colchicines (Col), amiloride (AMR), FITC, 4\% (v/v) formaldehyde, Na-Flu salt, Triton X-100, and 4',6-diamidino-2phenylindole (DAPI) were obtained from Sigma-Aldrich (St Louis, MO, USA). Primary polyclonal rabbit anti-human zonula occludens-1 (ZO-1) antibody and Alexa Fluor-488 goat anti-rabbit secondary antibody were purchased from Thermo Fisher Scientific. Dimethyl sulfoxide (DMSO) was purchased from Amresco Inc. (Solon, OH, USA); dimethylformamide, acetone, pyridine, acetic anhydride, dibutyltin dilaurate (DBTDL), and other reagents were of reagent grade and obtained from commercial sources.

\section{Methods}

\section{Synthesis and characterization of PA-FITC}

The synthetic route of PA was described in our previous report. ${ }^{16}$ PA-FITC was prepared by the method of Jiang et al. ${ }^{17}$ PA (100 mg) was dissolved in $3 \mathrm{~mL}$ of DMSO by stirring, and then, $10 \mathrm{mg}$ FITC was added. Pyridine $(400 \mu \mathrm{L})$ and DBTDL $(30 \mu \mathrm{L})$ were added to the above solution, and the mixture was subsequently stirred at $100^{\circ} \mathrm{C}$ for 4 hours. The tawny reactant was cooled to room temperature, precipitated with ethyl alcohol to remove free dye, and then washed with ethyl alcohol followed by acetone. The yellow flocculent solid material was obtained through filtering and dried under vacuum at $40^{\circ} \mathrm{C}$. The final product was identified by ${ }^{1} \mathrm{H}$ nuclear magnetic resonance ( ${ }^{1} \mathrm{H}$ NMR; Bruker Corporation, Billerica, MA, USA) spectrometry. The degree of substitution (DS) was defined as how many hydroxyl groups in each glucose unit of PA were substituted by FITC and was determined by ${ }^{1} \mathrm{H}$ NMR.

\section{Preparation and characterization of PA-FITC NPs}

PA-FITC NPs were prepared by dialysis as described previously. ${ }^{18}$ In brief, PA-FITC (10 mg) was dissolved in $2 \mathrm{~mL}$ of DMSO solution (DMSO/ $\left.\mathrm{H}_{2} \mathrm{O}=9: 1\right)$; then, the mixture was transferred to a preswollen dialysis membrane
(MO cutoff $=8-14 \mathrm{kDa}$; Beijing Solarbio Technology, Ltd., Beijing, People's Republic of China) and dialyzed against deionized water for 48 hours at room temperature (deionized water was exchanged at 2- to 3-hour intervals). The particle size and size distribution of the NPs were measured by dynamic light scattering (DLS; Zetasizer 2000; Malvern Instruments Ltd., Malvern, UK). The morphology of the PA-FITC NPs was observed by TEM (JEM100CXII; JEOL Ltd., Tokyo, Japan) and scanning electron microscopy (SEM; SUPRA 55VP; Carl Zeiss Microscopy GmbH, Jena, Germany). For TEM, a drop of diluted aqueous suspension of NPs was deposited on a copper grid and air-dried prior to observation. For SEM, a drop of the NP suspension was placed on a silicon wafer. After drying, the samples were coated with gold using an ion coater (EIKO, IB-3; EIKO, Tokyo, Japan).

\section{Cell culture}

Cells were cultured at $37^{\circ} \mathrm{C}$ with $5 \% \mathrm{CO}_{2}$ in a humidified atmosphere in supplemented DMEM-F12 with or without phenol red. ${ }^{8,9}$ At confluence, the cells were subcultured using trypsin-EDTA solution.

\section{CCK-8 cytotoxicity assay}

The in vitro cytotoxicity of the different concentrations of PA-FITC NPs $(15,30,60,125,250,500,1,000$, and $2,000 \mu \mathrm{g} / \mathrm{mL}$ ) was tested using CCK-8 viability assay. ${ }^{13}$ BeWo b30 cells were seeded at $1 \times 10^{4}$ cells/well in 96-well plates and incubated at $37^{\circ} \mathrm{C}$ and $5 \% \mathrm{CO}_{2}$ for 24 hours, followed by exposure to PA-FITC NPs. Cells with $0 \mu \mathrm{g} / \mathrm{mL}$ of NPs were used as negative control. After 48 hours of incubation, NPs in culture supernatant were removed, and the cells were washed three times in PBS before incubation with $10 \mu \mathrm{L}$ of CCK-8 premixed with $100 \mu \mathrm{L}$ of fresh cell culture medium (a total of $110 \mu \mathrm{L}$ was added to each well). After incubation for 2 hours, the absorbance was measured at $450 \mathrm{~nm}$ using a plate reader (Varioskan Flash; Thermo Fisher Scientific). The percentage of viable cells (CV\%) was calculated as follows: $\mathrm{CV} \%=$ optical density (test)/optical density (control) $\times 100 \%$.

\section{HCG cytokine assay}

Production of the placenta hormone HCG was estimated to assess cell functionality. ${ }^{13}$ BeWo b30 cells were seeded at $1 \times 10^{4}, 2 \times 10^{4}, 4 \times 10^{4}$, and $8 \times 10^{4}$ cells $/ \mathrm{mL}$ in 96 -well plates. Cytokine levels in cell-culture supernatants were measured using the HCG ELISA as outlined by the manufacturer after 2, 3, and 4-day culture. For the ELISA, HCG concentration in cell-culture supernatants was determined from the standard 
curve and expressed as protein-corrected values $(\mathrm{ng} / \mathrm{mL}$ of total protein).

\section{Uptake inhibition}

To study the effect of various inhibitors on the uptake of NPs, the cells were preincubated with the following inhibitors individually, at concentrations which were nontoxic to the cells: $7 \mu \mathrm{g} / \mathrm{mL}$ of CPZ to inhibit clathrin-mediated endocytosis, $50 \mu \mathrm{g} / \mathrm{mL}$ of $\mathrm{NY}$ to caveolae-mediated endocytosis, $10 \mu \mathrm{g} / \mathrm{mL}$ of Col to inhibit pinocytosis, and $50 \mu \mathrm{M}$ AMR to inhibit macropinocytosis. ${ }^{17,19,20}$ Following preincubation for 1 hour at $37^{\circ} \mathrm{C}$, the inhibitor solutions were removed, and the freshly prepared PA-FITC NPs $(250 \mu \mathrm{g} / \mathrm{mL})$, in medium containing inhibitors at the same concentrations, were added and incubated for a further 2 hours. Subsequently, the cells were washed three times with PBS; then, $200 \mu \mathrm{L}$ cell lysis solution $(0.25 \%$ Triton X-100) was added for 1 hour after which cultures were analyzed quantitatively using a microplate reader $(\lambda$ ex $=494 \mathrm{~nm}, \lambda$ em $=519 \mathrm{~nm})$. The group in the presence of PA-FITC NPs but without inhibitor treatment was used as the control, and their uptake was expressed as $100 \%$. Percentage uptake was calculated from the following formula: inhibition uptake $\%=(1-$ optical density [test] $/$ optical density [control] $) \times 100 \%$.

\section{Monolayer characterization}

BeWo b30 cells were seeded onto Transwell membranes at $1 \times 10^{5}$ cells/well and incubated at $37^{\circ} \mathrm{C}$ with $5 \% \mathrm{CO}_{2}$. The development of the monolayer was monitored using TEER measurements followed by Na-Flu transport on days 1-8 after seeding, as well as TEM and tight junction staining.

\section{TEER measurements}

Monolayer integrity was measured with a Millicell ERS-2 Volt-Ohm meter and accessories (Millipore Corp.) containing $1.5 \mathrm{~mL}$ of medium at room temperature. TEER values for the cell layer were obtained by subtracting the intrinsic resistance (blank insert membrane) from the total resistance (insert membrane with cells). TEER values were corrected for surface area $\left(1.12 \mathrm{~cm}^{2}\right)$ and expressed as $\Omega \cdot \mathrm{cm}^{2}$.

\section{Na-Flu transport}

Cell confluence was determined by retardation of Na-Flu transport across the monolayer. A $0.5 \mathrm{~mL}$ aliquot of $5 \mu \mathrm{M} \mathrm{Na}-\mathrm{Flu}$ was added to the apical chamber, and $1.5 \mathrm{~mL}$ of DMEM-F12 (without phenol red) was added to the basal chamber of each Transwell. Plates were incubated for 30 minutes, and then,
$50 \mu \mathrm{L}$ samples were removed in triplicate from the basal chamber, transferred to a black 96-well plate, and read as described previously at each sampling time point.

\section{TEM}

BeWo b30 cells grown on insert membranes were fixed and processed according to Bode et al. ${ }^{21}$ After ultrathin sectioning, the samples were analyzed by TEM (JEM100CXII; JEOL Ltd.).

\section{Tight junction staining}

To quantify the integrity of the confluent cell monolayer, immunostaining of tight junctions was performed on day 6 postseeding (PS). BeWo b30 cells grown on Transwell membranes were fixed in $2 \%(\mathrm{v} / \mathrm{v})$ paraformaldehyde at $4{ }^{\circ} \mathrm{C}$ for 30 minutes, washed in PBS, and permeabilized with $0.25 \%$ Triton X-100 for 20 minutes at room temperature. The cells were incubated with a blocking agent $(3.5 \%$ bovine serum albumin in PBS) for 1 hour at room temperature. Primary rabbit anti-ZO-1 was diluted (1:100) in blocking agent $(2 \%$ bovine serum albumin in PBS) and incubated with the cells for 18 hours at $4^{\circ} \mathrm{C}$. Secondary antibody (1:200 dilution in PBS) was incubated with the cells for 20 minutes at room temperature in the dark. Membranes were incubated with DAPI and mounted on glass slides. Staining was observed using a confocal laser scanning microscope (LSM 710; Carl Zeiss Microscopy GmbH). Imaging parameters were selected to optimize resolution, and images were acquired with Leica confocal software (Leica Microsystems Ltd., Wetzlar, Germany).

\section{Transport of PA-FITC NPs}

Transport studies were conducted using BeWo b30 cells following the protocol of Cartwright et $\mathrm{al}^{8}$ and Kloet et al. ${ }^{22}$ BeWo b30 cells were seeded at $1 \times 10^{5}$ cells/well in Transwell and grown to confluence as described above, using the optimized protocol for PA-FITC NPs. Experiments were conducted in supplemented DMEM-F12 without phenol red. Transport of PA-FITC NPs across the BeWo cell monolayer was carried out under standard cell culture conditions. Inhibitors were used at the same concentrations as in the uptake test. At t0, $0.6 \mathrm{~mL}$ of $250 \mu \mathrm{g} / \mathrm{mL}$ NP dispersion $\left(223.2 \mu \mathrm{g} / \mathrm{cm}^{2}\right)$ was added to the apical chamber. For imaging analysis, the cells were exposed to PA-FITC NPs for 24 hours at day 6 PS. Transwell membranes were fixed in 4\% formaldehyde, incubated sequentially with phalloidin and DAPI for 40 and 5 minutes, respectively, and then mounted on glass slides. Staining was observed using a Zeiss LSM 780 
confocal system. NPs transported through the BeWo b30 cell monolayer were characterized by SEM.

\section{Statistical analysis}

Data are presented as mean \pm SD. Statistical significance was determined by one-way analysis of variance, and $P$-values $<0.05$ were considered significant.

\section{Results}

\section{Synthesis and characteristics of PA-FITC}

In this study, PA (with the substitution of 2.6 acetyl groups per one hydroglucose unit of pullulan) was used to prepare PA-FITC. Figure 1 shows the ${ }^{1} \mathrm{H}$ NMR spectra of PA and PA-FITC in DMSO-d $\mathrm{d}_{6}$. Hydroxyl proton signals, observed at 4.5-5.6 ppm in the ${ }^{1} \mathrm{H}$ NMR spectrum of pullulan (Figure 1B), and methyl proton signals, observed at 1.8-2.2 ppm, which are assigned to the acetyl groups, appeared in the ${ }^{1} \mathrm{H}$ NMR spectrum of PA (Figure 1B). In the ${ }^{1} \mathrm{H}$ NMR spectrum of PA-FITC (Figure 1A), the prominent peaks of FITC were observed at $6.0-8.5$ and $10.0-10.2 \mathrm{ppm}$. The following proton signals were visible on the basis of the protocol: 2.60-4.60 (glucose $2 \mathrm{H}, 3 \mathrm{H}, 4 \mathrm{H}, 5 \mathrm{H}$, and $6 \mathrm{H}$ ), 4.60 (glucose $1 \mathrm{H} \alpha_{(1-6)}$ ), 5.05 (glucose $1 \mathrm{H} \alpha_{(1-4)}$ ), and 4.60-5.57 (glucose, $\left.-\mathrm{OH}\right){ }^{23}$ The DS was calculated from the equation: $\mathrm{DS} \%=\left(\mathrm{A}_{\delta 10.16}\right) /$ $\left(\mathrm{A}_{\delta 4.60}+\mathrm{A}_{\delta 5.05}\right) \times 100 \%$. Under the optimized reaction conditions, the DS of FITC was $16.10 \%$.

\section{Characterization of PA-FITC self-assembled NPs}

Figure 2 shows that PA-FITC NPs were spherical in shape with a solid structure and almost uniformly sized. The morphology of these particles was in accordance with

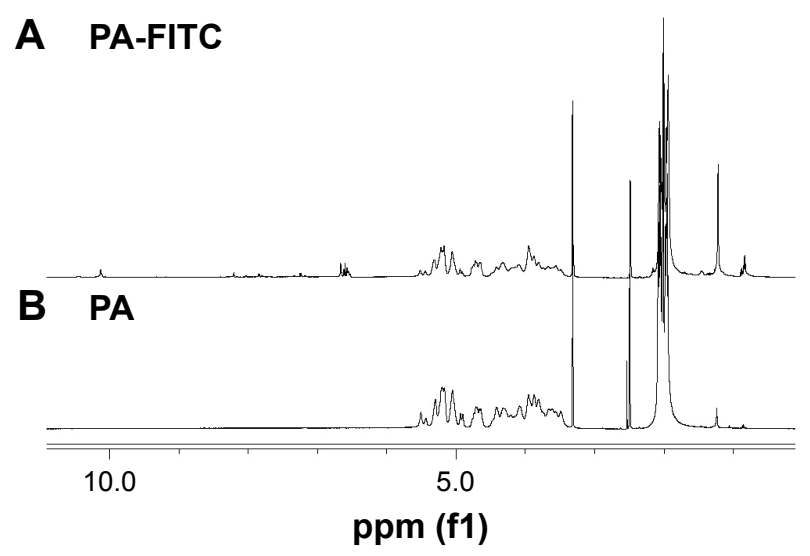

Figure I 'H NMR spectra of materials in DMSO-d6.

Note: (A) 'H NMR spectra of PA-FITC; (B) 'H NMR spectra of PA.

Abbreviations: DMSO-d6, dimethylsulfoxide-d6; 'H NMR, 'H nuclear magnetic resonance; PA-FITC, fluorescein isothiocyanate-conjugated pullulan acetate.
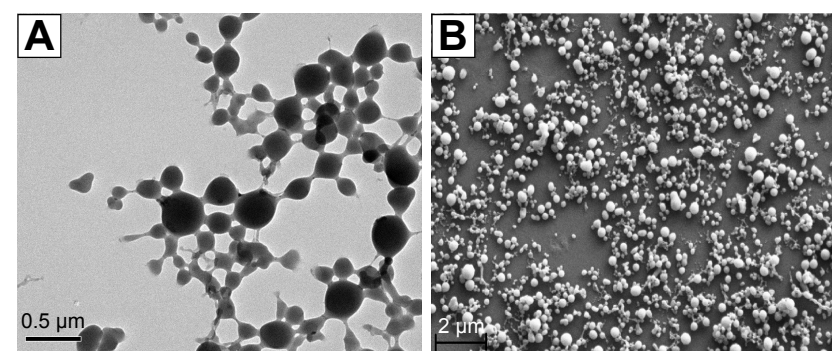

Figure 2 Morphology of PA-FITC NPs prepared by the dialysis method. Notes: (A) TEM image of NPs; (B) SEM image of NPs.

Abbreviations: PA-FITC NPs, fluorescein isothiocyanate-conjugated pullulan acetate nanoparticles; SEM, scanning electron microscopy; TEM, transmission electron microscopy.

previous literature describing self-assembled amphiphilic polymeric micelles. The average diameter of these NPs was $283.7 \pm 3.5 \mathrm{~nm}(\mathrm{n}=3)$, and the size distribution was narrow as evaluated by DLS. In addition, the $\zeta$ potential was $-18.3 \pm 7.4 \mathrm{mV}(\mathrm{n}=3)$, which indicates good stability in aqueous solution.

\section{CCK-8 cytotoxicity assay}

Figure 3 shows that PA-FITC NPs did not exhibit significant toxicity over a large range of concentrations after 48 hours of incubation. To our surprise, the cell viability of BeWo b30 cells was up to $100 \%$ even at the highest concentration of $2,000 \mu \mathrm{g} / \mathrm{mL}$, which demonstrated the good safety of the NPs.

\section{HCG assay}

HCG is a cytokine produced by the placenta. The release of HCG from BeWo b30 cells after 2, 3, and 4 days was

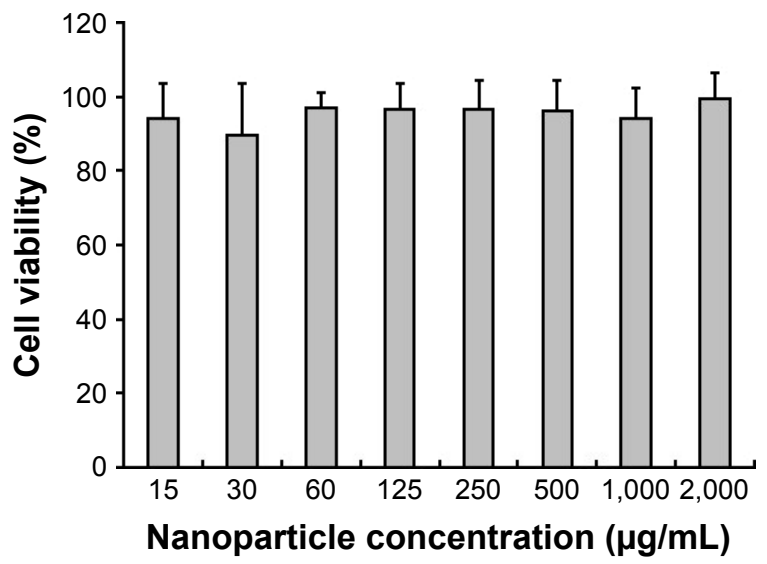

Figure 3 Cell viability of BeWo b30 cells exposed to PA-FITC NPs for 48 hours as measured by CCK-8 assay.

Note: Each value represents the mean \pm SD $(n=8)$.

Abbreviations: CCK-8, Cell Counting Kit-8; PA-FITC NPs, fluorescein isothiocyanate-conjugated pullulan acetate nanoparticles. 


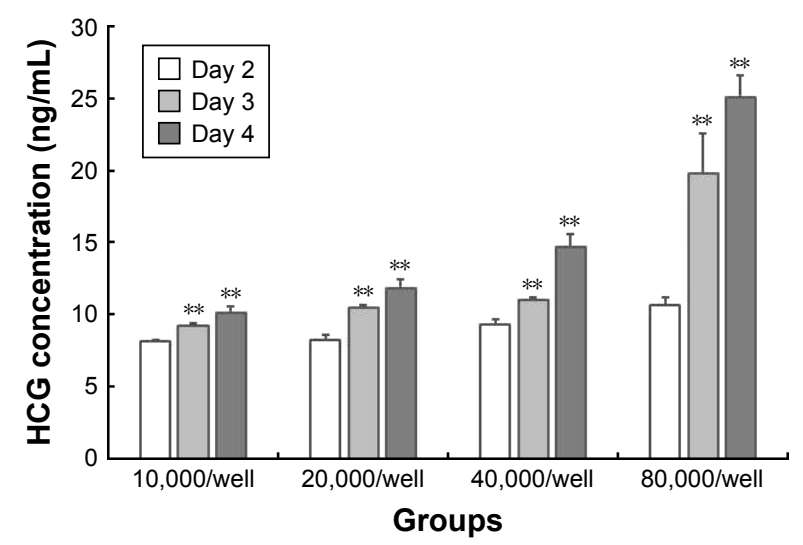

Figure 4 HCG release from BeWo b30 cells at 2, 3, and 4 days as determined by ELISA.

Notes: Data are represented as mean $\pm S D(n=4) . * * p<0.01$ vs day 2 .

Abbreviations: ELISA, enzyme-linked immunosorbent assay; HCG, human chorionic gonadotropin.

determined using an ELISA (Figure 4). The concentration of HCG increased as the cell number increased from 10,000 to 80,000 per well. In addition, the concentration of $\mathrm{HCG}$ increased when cultivated for a longer time $(P<0.01)$. The results showed that the BeWo b30 cells had good physiological function.

\section{Uptake inhibition}

As shown in Figure 5, the cellular uptake of PA-FITC NPs was reduced significantly $(P<0.01)$ in comparison with the control group, as detected by fluorescence intensity. The inhibition rates of NY, Col, AMR, and CPZ were 55\%, 51\%, $24.2 \%$, and $23.9 \%$, respectively. The results indicated that PA-FITC NPs were mainly taken up into cells by caveolaemediated endocytosis and pinocytosis.

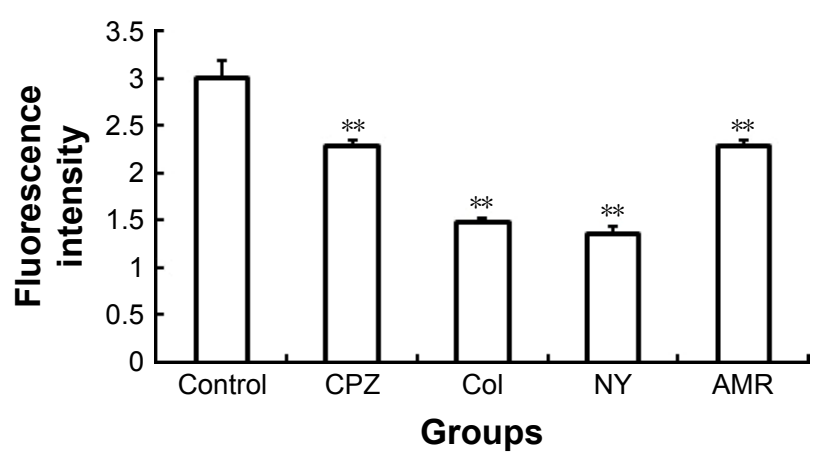

Figure 5 Effect of endocytic inhibitors on the internalization of PA-FITC NPs, evaluated by fluorescence intensity.

Notes: Data are represented as mean $\pm S D(n=6) . * * P<0.0$ I vs control.

Abbreviations: AMR, amiloride; Col, colchicine; CPZ, chlorpromazine; NY, nystatin; PA-FITC NPs, fluorescein isothiocyanate-conjugated pullulan acetate nanoparticles.

\section{Monolayer confirmation}

TEER and Na-Flu transport data (Figure 6) verified that a BeWo b30 cell monolayer was formed over 5 days, with a steady increase in TEER values and Na-Flu retardation. TEER values between 30 and $60 \Omega \cdot \mathrm{cm}^{2}$ were considered acceptable for performing transport studies. ${ }^{12}$ Moreover, antibody staining for the tight junction protein ZO-1 was performed on day 6 PS to examine barrier integrity in the confluent cell monolayer. DAPI counterstaining was used to visualize the nuclei. The presence of the tight junction protein ZO-1 was observed (Figure 7A), which lends support to the progression of confluent monolayer formation indicated in the TEM images. TEM images on day 6 PS revealed a confluent monolayer with visible tight junctions (Figure 7B) and villus structure (Figure 7C).

\section{Transport of PA-FITC NPs}

PC Transwell membranes with $3 \mu \mathrm{m}$ pore size were chosen for model optimization. BeWo b30 cells were seeded at $1 \times 10^{5}$ cells/well. The monolayer was monitored on day 6 PS using TEER. PA-FITC NP dispersion was added to the apical chamber, and after 24 hours, PA-FITC NPs were found in the cytoplasm of cells on the Transwell membranes (Figure 8A-D), while some NPs added to the apical chamber of the cell monolayer were found in the basal chamber (Figure 8E). The results showed that the PA-FITC NPs were capable of transplacental transport.

\section{Discussion}

Nanomedicines offer unique advantages, such as sustained, controlled release and targeting of specific cells or tissues. ${ }^{14}$ No nanomedicine has been approved for use in pregnant women, however, and the clinical need is tremendous. It is essential to achieve a comprehensive insight into the transplacental transport mechanisms before clinical application. The aim of this work was to evaluate the safety and to elucidate the possible mechanism of PA NPs in crossing the placenta.

In the present study, PA-FITC NPs did not show significant toxicity to BeWo b30 cells at concentrations of $15-2,000 \mu \mathrm{g} / \mathrm{mL}$ (Figure 3), indicating the good biocompatibility of pullulan-derived NPs. The result was the same as reported in our previous work. ${ }^{16} \mathrm{HCG}$, a hormone of trophoblastic origin, was considered to be a marker of placental trophoblast differentiation. ${ }^{24}$ The concentrations of HCG increased in the supernatants of the cell cultures with increasing numbers of cells and culture time (Figure 4), 

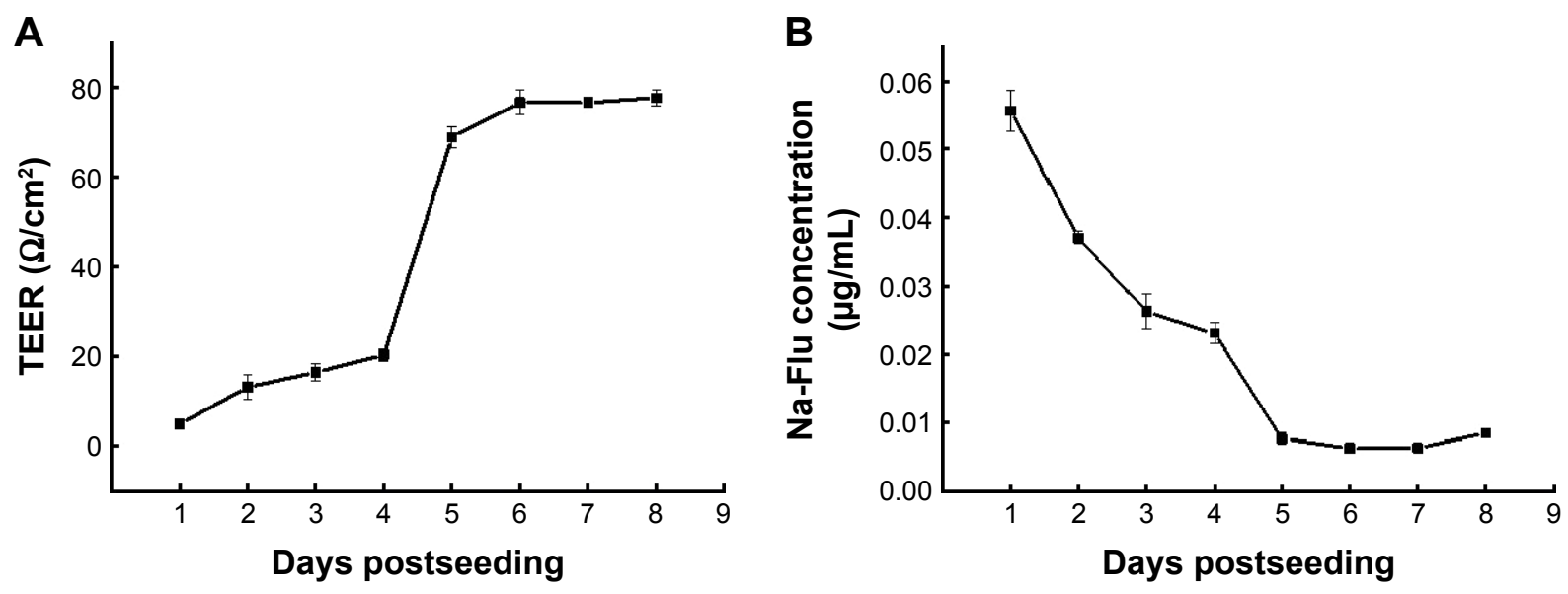

Figure 6 Formation of monolayers measured using TEER and Na-Flu transport.

Notes: $(A)$ TEER values of cells postseeding $(n=4)$; $(B)$ concentration of Na-Flu transport $(n=6)$. Data are represented as mean \pm SD.

Abbreviations: $\mathrm{Na}$-Flu, fluorescein sodium; TEER, transepithelial electrical resistance.

which demonstrated the good physiological function of BeWo b30.

The endocytic pathway of PA-FITC NPs in BeWo b30 cells was characterized. NY, a highly selective inhibitor of caveolae-mediated transport processes, showed the best inhibitory effect (Figure 5). Col also obviously affected NP uptake by the BeWo b30 cells, with an inhibition rate of $51 \%$. The uptake study showed that caveolae-mediated endocytosis and pinocytosis may be the main mechanisms involved in the internalization of PA-FITC NPs, with endocytosis being the most important. Endocytosis of NPs by the placenta is important in that it can alter the transplacental passage of drugs. ${ }^{3,4,25}$

The BeWo b30 cell line is considered an established model of the human placental barrier. These cells can form polarized monolayers useful for studying transplacental transport. ${ }^{3}$ The integrity of the BeWo cell monolayers on days 1-8 PS was investigated by daily TEER measurements in conjunction with transport studies involving the paracellular tracer Na-Flu (Figure 6). By day 5 PS, the apparent permeability of Na-Flu had reached a minimal plateau, and the TEER values were in the reported desirable range. ${ }^{12}$ This meant that, by day 5 PS, the cells had formed tight junctions, which limited passive permeation. This is in agreement with published studies. ${ }^{8,11,12}$

The Transwell matrix is commonly used for transport studies across cellular barriers. ${ }^{8,22}$ Confocal and TEM images (Figure 7) provided visual confirmation to validate this model. Tight junctions could be seen 6 days PS, together with the increased TEER values and decreased Na-Flu transport data, indicating a suitable opportunity for the study of transport over the subsequent 48 hours. Confocal images of NP
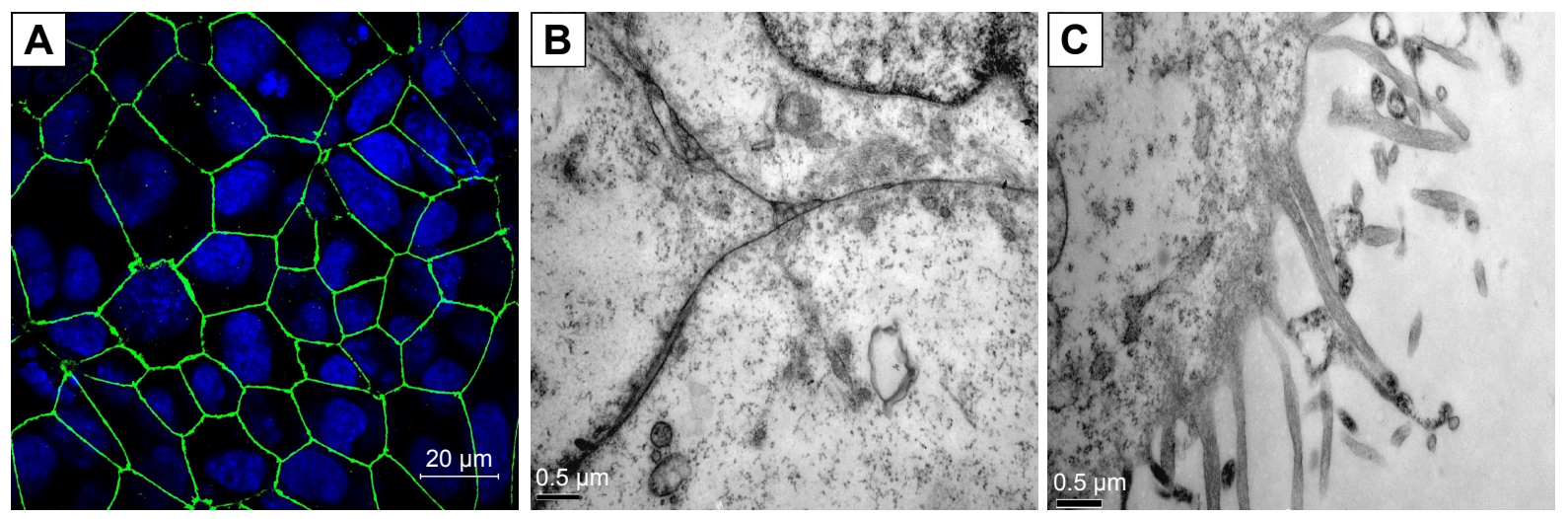

Figure 7 Confocal and TEM images of BeWo b30 cells.

Notes: BeWo b30 cells were grown on PC membranes with $3 \mu \mathrm{m}$ pore size. Cell nuclei were stained with DAPI (blue) and tight junctions with ZO-I (green) (A). Monolayer formation and microvilli structure: tight junctions (B) and villus structure $(\mathbf{C})$.

Abbreviations: DAPI, 4',6-diamidino-2-phenylindole; PC, polycarbonate; TEM, transmission electron microscopy; ZO-I, zonula occludens-I. 

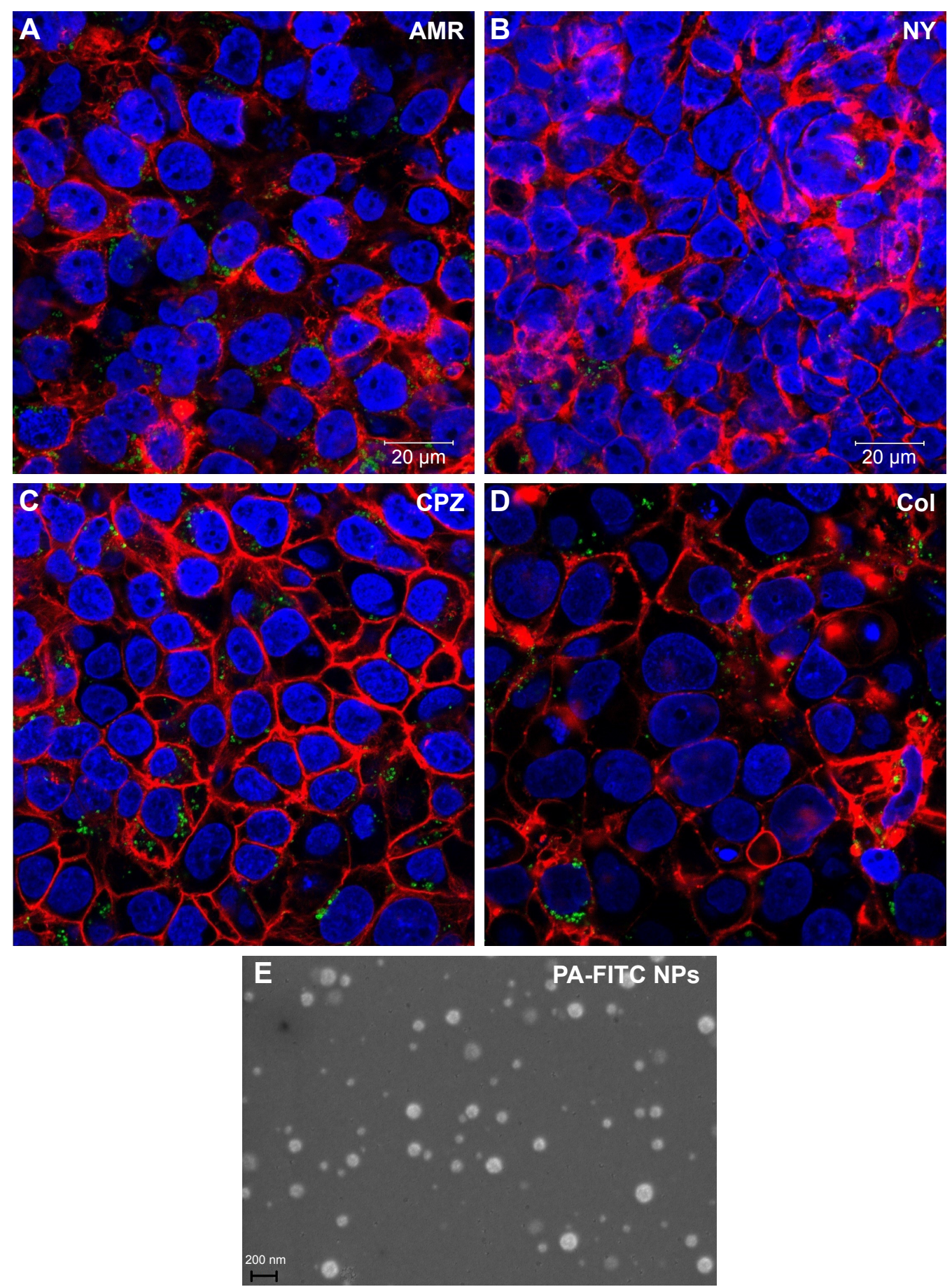

Figure 8 Confocal imaging of BeWo b30 cells and morphological characteristics of PA-FITC NPs. Transwell ${ }^{\circledR}$ cultures were treated with the endocytic inhibitors AMR, NY, CPZ, or Col.

Notes: Cell nuclei were stained with DAPI (blue), and cytoskeleton was stained with phalloidin (red), while PA-FITC NPs (green) can be seen in the cytoplasm (A-D). (E) PA-FITC NPs after transportation across the membrane, determined by SEM of the NY group, which is representative of all groups.

Abbreviations: AMR, amiloride; Col, colchicine; CPZ, chlorpromazine; DAPI, 4',6-diamidino-2-phenylindole; mag, magnification; NY, nystatin; PA-FITC NPs, fluorescein isothiocyanate-conjugated pullulan acetate nanoparticles; SEM, scanning electron microscopy.

localization within the monolayer (Figure 8A-D) suggest that the particles may take a transcellular route through the monolayer. As shown in Figure 8E, some NPs in the basal chamber were transported from the apical chamber, demonstrating that the PA-FITC NPs showed translocation ability.
Caveolae-mediated endocytosis may be the primary pathway during PA-FITC NP uptake into cells and transplacental transport. Caveolae have been implicated in numerous functions, including membrane trafficking via endocytosis and transcytosis. ${ }^{25}$ Caveolin-1, a major protein of the caveolae family, 
was originally reported as "caveolin," the 22-kDa protein first discovered in the early 1990s. The caveolin-1 signaling pathway is known for its role in regulating cellular functions, including signal transduction and endocytosis. ${ }^{26}$ Zhang et $\mathrm{al}^{27}$ prepared PLGA NPs encapsulating coumarin-6 and analyzed the endocytic mechanism of NPs through this membrane interface by means of various endocytic inhibitors. The results showed that NPs were internalized predominantly via macropinocytosis and caveolin-mediated endocytic pathways. Rattanapinyopituk et $\mathrm{al}^{4}$ determined the possible translocation pathway of gold NPs across the maternal-fetal barrier in pregnant Institute of Cancer Research (ICR) mice. The mice were intravenously injected with $0.01 \%$ of 20 - and 50 -nm gold NP solutions on days 16 and 17 of gestation. The study suggested that intravenous administration of gold NPs may upregulate clathrin- and caveolin-mediated endocytosis at the maternal-fetal barrier in mouse placenta. Caveolin-1 is a key negative regulator of endothelial nitric oxide synthase (eNOS) activation. ${ }^{28}$ Under physiological conditions, caveolin-1 can bind to eNOS, leading to eNOS inhibition and reduced NO production..$^{29}$ eNOS binds to the caveolin-1 scaffolding domain and remains inactive when bound. When the interaction is disrupted, eNOS is active, which leads to increases in NO level and vascular permeability. ${ }^{30}$ Consequently, caveolin-1 may regulate translocation through the caveolin-1/eNOS pathway. By combining the result with the endocytosis mechanism, we may find a good way to inhibit transplacental transport of nanodrugs. As shown in Figure 4, NY markedly inhibits the uptake of PA NPs by BeWo b30 cells, meaning that NY or other caveolin-1 inhibitors may decrease the quantity of transplacental transport, prolonging the half-life time in maternal circulation of a nanodrug. We plan to investigate this mechanism further in our future research.

\section{Conclusion}

PA-FITC NPs are nontoxic to BeWo b30 cells, can cross the Transwell placental barrier model, and are mainly internalized through caveolae-mediated endocytosis and pinocytosis pathways, mostly via the former pathway. Our data could contribute to the improvement of selective uptake strategies and transplacental transport for nanomedicines. Our current studies mainly addressed whether or not NPs are able to cross the placental barrier. However, the in-depth transport mechanisms and possible adjustment methods underlying NP translocation across the placenta are still unknown and will be the subject of our future research.

\section{Acknowledgments}

Hongbo Tang and Ziwen Jiang are co-first authors. All the authors thank Professor Erik Rytting (University of Texas
Medical Branch) for donating the choriocarcinoma cell line BeWo b30. This work was financially supported by the National Key Research and Development Program of China (grant number: 2016YFC1000101), the National Natural Science Foundation of China (grant number: 81301322) and Beijing Obstetrics and Gynecology Hospital, Capital Medical University (fcyy201707).

\section{Disclosure}

The authors report no conflicts of interest in this work.

\section{References}

1. Koopaei NN, Abdollahi M. Opportunities and obstacles to the development of nanopharmaceuticals for human use. Daru. 2016;24:25.

2. Bobo D, Robinson KJ, Islam J, Thurecht KJ, Corrie SR. Nanoparticlebased medicines: a review of FDA-approved materials and clinical trials to date. Pharm Res. 2016;33(10):2373-2387.

3. Al-Enazy S, Ali S, Albekairi N, El-Tawil M, Rytting E. Placental control of drug delivery. Adv Drug Deliv Rev. 2017;116:63-72.

4. Rattanapinyopituk K, Shimada A, Morita T, et al. Demonstration of the clathrin- and caveolin-mediated endocytosis at the maternal-fetal barrier in mouse placenta after intravenous administration of gold nanoparticles. J Vet Med Sci. 2014;76(3):377-387.

5. Myllynen PK, Loughran MJ, Howard CV, Sormunen R, Walsh AA, Vähäkangas KH. Kinetics of gold nanoparticles in the human placenta. Reprod Toxicol. 2008;26(2):130-137.

6. Kolhar P, Mitragotri S. Polymer microparticles exhibit size and shape dependent accumulation around the nucleus after endocytosis. Adv Funct Mater. 2012;22(18):3759-3764.

7. Braakhuis HM, Kloet SK, Kezic S, et al. Progress and future of in vitro models to study translocation of nanoparticles. Arch Toxicol. 2015; 89(9):1469-1495.

8. Cartwright L, Poulsen MS, Nielsen HM, et al. In vitro placental model optimization for nanoparticle transport studies. Int J Nanomedicine. 2012;7:497-510.

9. Correia Carreira S, Walker L, Paul K, Saunders M. The toxicity, transport and uptake of nanoparticles in the in vitro BeWo b30 placental cell barrier model used within NanoTEST. Nanotoxicology. 2015; 9(Suppl 1):66-78.

10. Ali H, Kalashnikova I, White MA, Sherman M, Rytting E. Preparation, characterization, and transport of dexamethasone-loaded polymeric nanoparticles across a human placental in vitro model. Int $J$ Pharm. 2013;454(1):149-157.

11. Lopalco A, Ali H, Denora N, Rytting E. Oxcarbazepine-loaded polymeric nanoparticles: development and permeability studies across in vitro models of the blood-brain barrier and human placental trophoblast. Int J Nanomedicine. 2015;10:1985-1996.

12. Albekairi NA, Al-Enazy S, Ali S, Rytting E. Transport of digoxin-loaded polymeric nanoparticles across BeWo cells, an in vitro model of human placental trophoblast. Ther Deliv. 2015;6(12):1325-1334.

13. Grafmueller S, Manser P, Diener L, et al. Bidirectional transfer study of polystyrene nanoparticles across the placental barrier in an ex vivo human placental perfusion model. Environ Health Perspect. 2015; 123(12):1280-1286.

14. Muoth C, Aengenheister L, Kucki M, Wick P, Buerki-Thurnherr T. Nanoparticle transport across the placental barrier: pushing the field forward! Nanomedicine (Lond). 2016;11(8):941-957.

15. Singh RS, Kaur N, Rana V, Kennedy JF. Pullulan: a novel molecule for biomedical applications. Carbohydr Polym. 2017;171:102-121.

16. Tang HB, Li L, Chen H, et al. Stability and in vivo evaluation of pullulan acetate as a drug nanocarrier. Drug Deliv. 2010;17(7):552-558.

17. Jiang L, Li X, Liu L, Zhang Q. Cellular uptake mechanism and intracellular fate of hydrophobically modified pullulan nanoparticles. Int J Nanomedicine. 2013;8:1825-1834. 
18. Tang H, Feng X, Zhang T, et al. Stability, pharmacokinetics, biodistribution and safety assessment of folate-conjugated pullulan acetate nanoparticles as cervical cancer targeted drug carriers. J Nanosci Nanotechnol. 2015;15(9):6405-6412.

19. Wang H, Wu L, Reinhard BM. Scavenger receptor mediated endocytosis of silver nanoparticles into J774A.1 macrophages is heterogeneous. ACS Nano. 2012;6(8):7122-7132.

20. Plummer EM, Manchester M. Endocytic uptake pathways utilized by CPMV nanoparticles. Mol Pharm. 2013;10(1):26-32.

21. Bode CJ, Jin H, Rytting E, Silverstein PS, Young AM, Audus KL. In vitro models for studying trophoblast transcellular transport. Methods Mol Med. 2006;122:225-239.

22. Kloet SK, Walczak AP, Louisse J, et al. Translocation of positively and negatively charged polystyrene nanoparticles in an in vitro placental model. Toxicol in Vitro. 2015;29(7):1701-1710.

23. Yang WZ. Preparation of self-aggregated nanoparticles of cholesterolbearing pullulan derivatives and the primary study on using them as the novel carriers of drugs [dissertation]. Peking Union Medical College, 2009.

24. Juch H, Nikitina L, Reimann S, et al. Dendritic polyglycerol nanoparticles show charge dependent bio-distribution in early human placental explants and reduce hCG secretion. Nanotoxicology. 2018;12(2): 90-103.
25. Sohn J, Brick RM, Tuan RS. From embryonic development to human diseases: the functional role of caveolae/caveolin. Birth Defects Res C Embryo Today. 2016;108(1):45-64.

26. Tu F, Pang Q, Chen X, Huang T, Liu M, Zhai Q. Angiogenic effects of apigenin on endothelial cells after hypoxia-reoxygenation via the caveolin-1 pathway. Int J Mol Med. 2017;40(6):1639-1648.

27. Zhang L, Xu Y, Cao W, Xie S, Wen L, Chen G. Understanding the translocation mechanism of PLGA nanoparticles across round window membrane into the inner ear: a guideline for inner ear drug delivery based on nanomedicine. Int J Nanomedicine. 2018;13:479-492.

28. Bandara N, Gurusinghe S, Lim SY, et al. Molecular control of nitric oxide synthesis through eNOS and caveolin-1 interaction regulates osteogenic differentiation of adipose-derived stem cells by modulation of Wnt/ $\beta$-catenin signaling. Stem Cell Res Ther. 2016;7(1):182.

29. Hofmann A, Gosemann JH, Takahashi T, Friedmacher F, Duess JW, Puri P. Imbalance of caveolin-1 and eNOS expression in the pulmonary vasculature of experimental diaphragmatic hernia. Birth Defects Res $B$ Dev Reprod Toxicol. 2014;101(4):341-346.

30. Sahay G, Alakhova DY, Kabanov AV. Endocytosis of nanomedicines. J Control Release. 2010;145(3):182-195.
International Journal of Nanomedicine

\section{Publish your work in this journal}

The International Journal of Nanomedicine is an international, peerreviewed journal focusing on the application of nanotechnology in diagnostics, therapeutics, and drug delivery systems throughout the biomedical field. This journal is indexed on PubMed Central, MedLine, CAS, SciSearch ${ }^{\circledR}$, Current Contents ${ }^{\circledR} /$ Clinical Medicine,

\section{Dovepress}

Journal Citation Reports/Science Edition, EMBase, Scopus and the Elsevier Bibliographic databases. The manuscript management system is completely online and includes a very quick and fair peer-review system, which is all easy to use. Visit http://www.dovepress.com/ testimonials.php to read real quotes from published authors. 